\title{
HVMANITAS
}

\section{Nuevas perspectivas de estudio de las tragedias de Séneca}

Autor(es): $\quad$ López, Aurora; Pociña, Andrés

Publicado por: Faculdade de Letras da Universidade de Coimbra, Instituto de Estudos

URL

persistente:

Clássicos

DOI: $\quad$ DOI:http://dx.doi.org/10.14195/2183-1718_63_16

Accessed : $\quad$ 26-Apr-2023 16:29:25

A navegação consulta e descarregamento dos títulos inseridos nas Bibliotecas Digitais UC Digitalis, UC Pombalina e UC Impactum, pressupõem a aceitação plena e sem reservas dos Termos e Condições de Uso destas Bibliotecas Digitais, disponíveis em https://digitalis.uc.pt/pt-pt/termos.

Conforme exposto nos referidos Termos e Condições de Uso, o descarregamento de títulos de acesso restrito requer uma licença válida de autorização devendo o utilizador aceder ao(s) documento(s) a partir de um endereço de IP da instituição detentora da supramencionada licença.

Ao utilizador é apenas permitido o descarregamento para uso pessoal, pelo que o emprego do(s) título(s) descarregado(s) para outro fim, designadamente comercial, carece de autorização do respetivo autor ou editor da obra.

Na medida em que todas as obras da UC Digitalis se encontram protegidas pelo Código do Direito de Autor e Direitos Conexos e demais legislação aplicável, toda a cópia, parcial ou total, deste documento, nos casos em que é legalmente admitida, deverá conter ou fazer-se acompanhar por este aviso. 
humanitas

Vol. LXIII

2011 


\title{
NUEVAS PERSPECTIVAS DE ESTUDIO DE LAS TRAGEDIAS DE SÉNECA ${ }^{1}$
}

\author{
Aurora López - Andrés Pociña \\ Universidad de Granada
}

"No sorriso dos olhos o sonho floresce em amanhã

E sortílega sempre acena nos alvores a estrada”

São versos fermosos do nosso muito caro amigo José Ribeiro Ferreira, que usurpamos para esta homenagem cordial e sincera

Aurora López e Andrés Pociña

\footnotetext{
${ }^{1}$ Esta publicación, desarrollada dentro del Proyecto de investigación FI200913693 FILO, subvencionado por el Ministerio de Ciencia e Innovación español, reproduce una conferencia dictada por la autora y el autor en el "Colóquio Poiesis em Latim", celebrado en el Centro de Estudos Clássicos e Humanísticos de la Universidad de Coimbra, el día 3 de junio de 2011. El peculiar desarrollo del tema se debe al deseo de dar a conocer la situación actual de la investigación conducente a la publicación de nuestro libro Séneca, Tragedias, perteneciente a un proyecto internacional sobre los cuatro grandes trágicos greco-romanos, dentro de un encuentro científico organizado por las catedráticas de Filología Griega de la Universidad de Coimbra Dra. Doña Maria do Céu Fialho y Dra. Doña Maria de Fátima Silva, a cargo de las cuales correrá la publicación del volumen correspondiente a las Tragedias de Eurípides. Deseamos que conste aquí nuestro agradecimiento por el trato espléndido que hemos recibido en el $\mathrm{CECH}$ de la Facultad de Letras de Coimbra, en esta ocasión y en las múltiples anteriores.
} 


\section{Resumen}

Con motivo de la preparación de un libro titulado Séneca, Tragedias, que formará parte de un conjunto de cuatro grandes monografías sobre el teatro de Esquilo, Sófocles, Eurípides y Séneca que elaboran coordinadamente clasicistas de las Universidades de Coimbra, Foggia, Granada y Valencia, la autora y el autor someten a debate una serie de aspectos fundamentales en el estudio de las Tragedias de Séneca. Se trata fundamentalmente de los temas básicos del tratamiento de la bibliografía, las últimas ediciones, la autenticidad de las tragedias, la representación, recitación o lectura de las piezas, la datación, la ideología y finalidad, la pervivencia y reescrituras.

Palabras clave: Séneca, Tragedias, algunos aspectos relevantes.

\section{Abstract}

The present paper aims to submit to discussion some aspects concerned with important themes of Senecan tragedies, derived from the actual composition of a book upon Séneca, Tragedias. The main arguments of discussion are concerned with bibliographic aspects, last editions, authenticity of the plays, problems of datation, influence of Senecan Tragedies in modern theaters, etc.

Key-words: Seneca, Tragedies, some discussed aspects.

\section{Presentación}

De los numerosos libros que hemos publicado conjuntamente sobre aspectos diversos del teatro grecorromano, sin duda el que nos produce mayor satisfacción es el gestado a lo largo de más de treinta años, que salió a la luz al fin, hace algo más de tres años, con el título de Comedia romana (Madrid, 2007) ${ }^{2}$. Fue un libro elaborado de forma muy intermitente, con períodos largos de redacción intensa de algunas partes, y con no menos frecuentes interrupciones, para dedicarnos a otros aspectos de nuestra investigación y tareas docentes. Sin embargo, el resultado parece confirmar nuestra creencia de que las obras que abarcan un contenido de muy amplio espectro, como puede ser el de los cuatro tipos de la comedia latina (palliata, togata, Atellana y mimo), en su gestación y en todo su desarrollo histórico, requieren un estudio reposado, una reflexión 2007.

${ }^{2}$ A. López - A. Pociña, Comedia romana, Tres Cantos, Ediciones Akal, 
prolongada, si se pretende hacer una obra mínimamente aceptable y de valía duradera. Iniciamos nuestra exposición con estas consideraciones porque se trata ahora de presentarles un nuevo libro, de límites menos amplios, pues se dedica exclusivamente a una parte de la producción literaria de un solo autor, en concreto a las Tragedias de Séneca, pero que representa una aportación fundamental en la construcción y desarrollo del teatro mundial. Nuestro deseo es doble: mostrarles el proyecto de nuestro trabajo, las importantes decisiones que a propósito del mismo ya hemos ido tomando, y ver la posibilidad de discutir con Ustedes las perspectivas de estudio que estamos poniendo en práctica en este nuevo atrevimiento nuestro.

Se trata ahora de un ambicioso proyecto que va más allá de nuestros estudios personales, pues abarca a los cuatro grandes tragediógrafos del Mundo Clásico, consistente en la realización de cuatro volúmenes independientes, pero de estructura y contenidos paralelos, elaborados en constante relación, dedicados cada uno de ellos a Esquilo, Sófocles, Eurípides y Séneca. En una fase posterior a la finalización de los cuatro volúmenes, los autores y las autoras que hemos trazado tan ambicioso proyecto, contemplamos la posibilidad de realizar en conjunto un volumen complementario, que sería una especie de introducción general a la Tragedia grecolatina. Diremos, en fin, que los filólogos y las filólogas embarcados en esta hermosa, pero también trabajosa y arriesgada, singladura somos:

Esquilo Prof. Dr. D. Francesco De Martino, Univ. de Foggia.

Sófocles Prof. Dr. D. José Vicente Bañuls Oller, Univ. de Valencia Prof $^{a}$. Dr ${ }^{\mathrm{a}}$. D ${ }^{\mathrm{a}}$. Carmen Morenilla Talens, Univ. de Valencia.

Eurípides $\operatorname{Prof}^{\mathrm{a}}$. Dra ${ }^{\mathrm{a}}$. ${ }^{\mathrm{a}}$. Maria do Céu Fialho, Univ. de Coimbra Prof $^{\mathrm{a}}$. Dr ${ }^{\mathrm{a}}$. D ${ }^{\mathrm{a}}$. Maria de Fátima Silva, Univ. de Coimbra.

Séneca, Tragedias Prof ${ }^{\mathrm{a}}$. Dra ${ }^{\mathrm{a}}$. Da . Aurora López López, Univ. de Granada

Prof. Dr. D. Andrés Pociña Pérez, Univ. de Granada.

Centrándonos, pues, en los aspectos de las Tragedias de Séneca que queremos presentar y someter a discusión, en la medida que el tiempo nos lo permita, se trata de los siguientes: 
1. La cuestión bibliográfica.

2. La cuestión ecdótica.

3. El corpus de las Tragedias de Séneca: el problema de la autenticidad.

4. Representación, recitación, lectura de las Tragedias.

5. El manido asunto de la datación de las Tragedias.

6. Ideología y finalidad de las Tragedias.

7. Reescrituras (solamente en los siglos XX y XXI).

\section{La cuestión bibliográfica}

Si hay un aspecto que asuste y ahuyente a cualquier estudiosa o estudioso que pretenda poner sus ojos en las Tragedias de Séneca, bien sea para la osadía de pechar con un acercamiento general al conjunto, pero incluso con el de una pieza determinada o hasta con un tema concreto, es, para decirlo con metáfora muy acertada de un antiguo profesor nuestro, la "enmarañada selva bibliográfica" a la que tendrá que enfrentarse previamente, siendo muy probable que en ella sucumban sus proyectos. En efecto, el tema filológico de los dramas del Cordobés resulta no sólo uno de los más problemáticos, más sembrado de cuestiones difíciles, intrincadas y controvertidas, sino, a pesar de tales inconvenientes, de los que han suscitado mayor interés y atracción en los estudios clásicos desde siempre, pero de forma especial incluso limitándonos a un panorama cronológico cercano al nuestro, esto es, el siglo XX y la década que ya va transcurrida del siglo XXI.

Dicho esto, y después de reconocer que no es lícito, sin grave riesgo, prescindir de obras clásicas sobre el asunto, pero que ya van quedando en cierto modo envejecidas y superadas por el paso del tiempo, consideramos que cualquier trabajo que pretendamos hacer sobre las Tragedias de Séneca no precisa ya repetir, una vez más y como se ha hecho hasta la saciedad, los planteamientos y desarrollos de incuestionablemente valiosas monografías, como pueden ser la aparentemente imprescindible Le théâtre de Sénèque (1924), de Léon Herrmann ${ }^{3}$, una obra que sigue comentándose una vez y otra vez, en muchas ocasiones para criticar posturas peculiares de su autor que, sin embargo, ya han sido una y mil veces criticadas; o, por poner otro ejemplo conspicuo, la no menos zarandeada obra Die Rezitationsdramen Senecas (1966), de Otto Zwierlein ${ }^{4}$, libro muy importante, pero al que no

\footnotetext{
${ }^{3}$ L. Herrmann, Le théâtre de Sénèque, Paris, Les Belles Lettres, 1924.

${ }^{4}$ O. Zwierlein, Die Rezitationsdramen Senecas. Mit einem kritisch-exege-
} 
se aporta nada nuevo poniendo todo énfasis en criticarlo o defenderlo una vez más, como si la idea central reflejada en el título fuese privativa de este autor, y nunca antes o después sustentada por otras personas, y como si no se tratase en ella más aspectos que el relativo al objetivo final de las piezas senecanas.

En suma, nuestro modo de proceder en el libro Séneca, Tragedias, en el apartado bibliográfico, aunque sólo fuera por el deseo de no tener que dedicarle decenas y decenas de páginas a una bibliografía que tenemos programada no como mera enumeración de autores y títulos, sino como crítica, consistirá en primer lugar en reenviar a nuestro posible público lector a algunos repertorios bibliográficos específicamente dedicados a Séneca trágico, que abarcan los estudios de un período general o bastante amplio, como son los publicados en Aufstieg und Niedergang ${ }^{5}$ por B. Seidensticker y D. Armstrong ${ }^{6}$ (años 1878-1978 y ss.), por O. Hiltbrunner ${ }^{7}$ (años 1965-1975), y por R. G. Tanner (filosofía y tradición en las tragedias) ${ }^{8}$, complementados para años posteriores por trabajos como el de A. Borgo ${ }^{9}$, y con la estupenda bibliografía general sobre Séneca, publicada en 1989 por A. L. Motto y J. R. Clark ${ }^{10}$. Y de forma incidental, dado que entre nuestras investigaciones en este campo A. Pociña recopiló, para el Congreso Internacional del Bimilenario de Séneca, celebrado en Córdoba, la bibliografía de todo tipo publicada sobre el autor en España a lo largo del siglo $\mathrm{XX}^{11}$, advertimos ya que en el largo período contemplado no existe ni un tischen Anhang (Meisenheim-am-Glan, Hain, 1966.

${ }^{5}$ Para fechas más antiguas no pueden olvidarse trabajos tan útiles como el de M. Coffey, "Seneca Tragedies including pseudo-Seneca Octavia and Epigrams attributed to Seneca. Report from the years 1922-1955", Lustrum 2 (1957) 113186.

${ }^{6}$ B. Seidensticker - D. Armstrong, "Seneca tragicus 1878-1978 (with Addenda 1979 ff.)", ANRW II 32,2 (1985) 916-968.

${ }^{7}$ O. Hiltbrunner, "Seneca als Tragödiendichter in der Forschung von 1965 bis 1975", ANRW II 32,2 (1985) 969-1051.

${ }^{8}$ R. G. Tanner, "Stoic Philosophy and Roman Tradition in Senecan Tragedy", ANRW II 32,2 (1985) 1100-1133.

${ }^{9}$ A. Borgo, "Alcuni recenti studi su Seneca tragico (1981-1993)", BStudLat 24 (1994) 584-606.

${ }^{10}$ A. L. Motto - J. R. Clark, Seneca. A Critical Bibliography 1900-1980. Scholarship on His Life, Thought, Prose, and Influence, Amsterdam, Hakkert, 1989.

${ }^{11}$ A. Pociña, "Los estudios sobre Séneca en España durante el siglo XX", 
solo volumen monográfico español sobre las Tragedias del Cordobés, ni buena ni mala ${ }^{12}$.

Naturalmente, recogeremos, por orden cronológico, y con la debida organización en secciones, la bibliografía fundamental publicada a partir de 1980. A modo de ejemplo, en la sección sobre las Tragedias en su conjunto, aparecerían, con una corta referencia crítica de sus principales contenidos y aportaciones, las siguientes monografías, ya analizadas en el estado actual de nuestra investigación:

A. J. Boyle (ed.), Seneca tragicus. Ramus essays on Senecan drama, Victoria, Aureal Publications, 1983.

N. T. Pratt, Seneca's Drama, Chapel Hill - London, Univ. of North Carolina Press, 1983.

F. Amoroso, Seneca, uomo di teatro?, Palermo, Palumbo, 1984.

G. Petrone, La scrittura tragica dell'irrazionale: note di lettura al teatro di Seneca. Palermo, Palumbo, 1984.

O. Zwierlein, Prolegomena zu einer kristischen Ausgabe der Tragödien Senecas, Wiesbaden, Abh. Akad. Wiss. Mainz, 1984.

J. D. Bishop, Seneca's Daggered Style. Political Code in the Tragedies, Meisenheim am Glan, Hain, 1985.

D. Henry, - E. Henry, The mask of power. Seneca's tragedies and imperial Rome, Warsminster, Wiltshire, Aris \& Phillips, 1985.

en AA. VV., Séneca dos mil años después, Córdoba, Universidad de Córdoba y Cajasur, 1997, pp. 739-774; trabajo completado y ampliado en "Bibliografía española sobre Séneca (años 1901-2000)", Florentia Iliberritana 17 (2006) 359410. Cf., para el mismo período, pero desde una perspectiva geográfica más amplia, E. Malaspina (ed.), Bibliografia senecana del XX secolo, Bologna, Pàtron, 2005.

${ }^{12}$ Algo semejante ocurre en el campo de estudios desarrollado en lengua portuguesa, en el que sólo encontramos las monografías de C. S. Vieira Gonçalves, Invectiva na tragédia de Séneca, Lisboa, Ed. Colibri, 2003, y en el Brasil la debida a la ilustre latinista Z. de A. Cardoso, Estudos sobre as tragédias de Sêneca, São Paulo, 2005, por lo que se refiere a libros, si bien no ignoramos aportaciones meritorias de la filología portuguesa, como los abundantes trabajos de J. A. Segurado e Campos, como "O simbolismo do fogo nas tragédias de Séneca", Euphrosyne 5 (1972) 185-247, “A linguagem dos gestos no teatro de Séneca”, Euphrosyne 15 (1987) 109-134; “Ratio e Voluntas no Pensamento de Séneca”, Classica 22 (1997) 79-92; “Séneca, Brecht e o Teatro épico", Classica 23 (1999) 9-26; o como los de M. C. Pimentel, “A conciliação da estética literária e da filosofia em Seneca: A tragédia Phaedra", Euphrosyne 15 (1987) 257-268; F. de Oliveira, "Imagem do Poder na Tragédia de Séneca", Humanitas 51 (1999) 49-83; etc. 
D. F. Sutton, Seneca on the Stage, Mnemosyne Suppl. 96, Leiden, Brill, 1986.

O. Zwierlein, Kritischer Kommentar zu den Tragödien Senecas, Stuttgart, Abh. Akad. Wiss. Mainz,1986.

T. F. Curley, The nature of Senecan drama, Roma, Ed. dell'Ateneo, 1986.

M. Billerbeck, Senecas Tragödien. Sprachliche und stilistische Untersuchungen, Leiden, 1988.

A. L. Motto - J. R. Clark, Senecan tragedy, Amsterdam, 1988.

M. Armisen-Marchetti, Sapientiae facies: étude sur les images de Sénèque, Paris, Les Belles Lettres, 1989.

T. G. Rosenmeyer, Senecan Drama and Stoic Cosmology, Berkeley, Univ. of California Press, 1989.

P. J. Davis, Shifting song: The chorus in Seneca's tragedies, Hildesheim, 1993.

F. Dupont, Les monstres de Sénèque. Pour une dramaturgie de la tragédie romaine, Paris, Éditions Belin, 1995.

L. Castagna (ed.), Nove studi sui cori tragici di Seneca, Milano, Aevum Antiquum, 1996.

A. J. Boyle, Tragic Seneca. An Essay in the Theatrical Tradition, London - New York, Routledge, 1997.

G. W. M. Harrison (ed.), Seneca in Performance, London, Duckworth, 2000.

G. Guastella, L'ira e l'onore: forme della vendetta nel teatro senecano e nella sua tradizione, Palermo, Palumbro, 2001.

A. Casamento, Finitimus oratori poeta. Declamazioni retoriche e tragedie senecane, Palermo, Flaccovio Editore, 2002.

C. S. Vieira Gonçalves, Invectiva na tragédia de Séneca, Lisboa, Ed. Colibri, 2003.

AA. VV., Sénèque le tragique. Huit exposés suivis de discussions, Vandoeuvres - Genève, Fondation Hardt, 2004.

J.-P. Aygon, Pictor in fabula. L'ecphrasis - descriptio dans les tragédies de Sénèque, Bruxelles, Éditions Latomus, 2004.

J. G. Fitch, Annaeana tragica. Notes on the text of Seneca's tragedies, Leiden, 2004.

C. A. J. Littlewood, Self-representation and illusion in Senecan tragedy, Oxford, Clarendon Press, 2004.

Z. de A. Cardoso, Estudos sobre as tragédias de Sêneca, São Paulo, Alameda Casa Editorial, 2005. 
F. Amoroso (ed.), Teatralità dei cori senecani, Palermo, Flaccovio Editore, 2006.

C. Kugelmeier, Die innere Vergegenwärtigung des Bühnenspiels in Senecas Tragödien, München, Beck, 2007.

\section{La cuestión ecdótica}

No es nuestra intención, ni aquí y ahora, ni en lugar o tiempo alguno, entrar de lleno en el multisecular y terriblemente enrevesado problema de la edición de las Tragedias de Séneca ${ }^{13}$; sin embargo, es obvio que quienes pretendemos escribir un nuevo estudio general sobre ellas no podemos marginar de nuestro interés la cuestión ecdótica, que ha realizado notables avances y ofrecido importantes novedades en las últimas décadas del siglo $\mathrm{XX}$.

Recordando sólo de pasada, para no hacer nuestra exposición de repente en un vacío, señalaremos que, en su línea esencial, las Tragedias se nos conservan en códices pertenecientes a dos familias, la representada esencialmente por el $\operatorname{cod}$. $E$, identificado de forma rápida con el nombre de códice Etrusco (Laurentianus 37, 13, saec. XI-XII), y la familia conocida como A, representada por una serie más numerosa de códices, de los que ocupan los lugares primordiales, siguiendo la ordenación y clasificación de Zwierlein, $P$ (Parisinus Latinus 8260, saec. XIII), $T$ (Parisinus Latinus 8031, saec. XV) y G (Exoniensis Bibl. Cathedralis 3549, saec. XIII) por un lado (hyparqu. $\square$ ), por otro $C$ (Cantabrigiensis, Corpus Christi College 406, saec. xiii), $S$ (Scorialensis T. III 11, saec. saec. XIII) y $V$ (Vaticanus Lat. 2829, saec. XIII) (hyparqu. $\square$ ). Sabido es que ambas familias presentan notables diferencias, que afectan no sólo a su antigüedad, valía ecdótica, ordenación y títulos de las tragedias, siendo además la familia $A$ la única que contiene el texto de la pretexta Octauia. Más adelante volveremos a referirnos a alguno de estos particulares.

Pues bien, en fecha tan cercana como 1986 la Universidad de Oxford publica, en su ya por entonces casi centenaria y siempre prestigiosísima Scriptorum Classicorum Bibliotheca Oxoniensis la muy anunciada y espe-

${ }^{13}$ Después de la edición oxoniense de Zwierlein, y de los diversos trabajos del editor que la precedieron, parece de justicia recordar sobre este aspecto el excelente trabajo de Margarethe Billerbeck, "Senecas Tragödientext und seiner Kritiker", en AA. VV., Sénèque le tragique. Huit exposés suivis de discussions, Vandoeuvres - Genève, Fondation Hardt, 2004, pp. 121-161. 
rada edición de las Tragedias elaborada por Otto Zwierlein ${ }^{14}$. Se echaba verdaderamente en falta en esta colección una parte tan significativa de las obras de Séneca ${ }^{15}$, un autor muy admirado en el Reino Unido y por la filología de expresión inglesa en general; el final de la prolongada espera se vio colmado con la aparición de una edición a todas luces excepcional, producto de muchos años de estudio sobre las Tragedias por parte de su responsable, y no sólo desde el punto de vista ecdótico, sino en forma de comentarios, estudios literarios, etc. ${ }^{16}$

La edición de Zwierlein ha supuesto, para decirlo de la forma más breve posible, una revolución total en los estudios de las Tragedias, según vamos a ver en los apartados siguientes de nuestra exposición. De momento, sin salirnos del campo de las ediciones, sin duda fue un elemento decisivo en el hecho de que la Collection des Universités de France, más familiarmente la "Colección Budé", retirase su ya muy añosa, pero sobre todo envejecida y superada en muchos aspectos, edición bilingüe en dos volúmenes, realizada por el siempre polémico latinista Léon Herrmann ${ }^{17}$. La nueva edición, llevada a cabo por François-Régis Chaumartin, es probablemente la más llamativa de las ediciones de la colección Budé aparecida en los últimos años: el editor comienza su "Introduction" como in medias res, dando una lista completa de las publicaciones de Zwierlein donde se contienen los principios adoptados para la colección Oxoniense, y a continuación resuelve en cuatro páginas mal organizadas todos los problemas de

${ }^{14}$ L. Annaei Senecae Tragoediae Incertorum auctorum Hercules [Oetaeus] Octauia recognouit breuique adnotatione critica instruxit Otto Zwierlein, Oxonii E Typographeo Clarendoniano Mcmlxxxvi.

${ }^{15}$ De hecho las Cartas morales a Lucilio ya habían sido editadas, en dos vols., en 1965, y los Diálogos en 1977, ambas ediciones al cuidado del prestigioso latinista oxoniense L. D. Reynolds.

${ }^{16}$ Cf. O. Zwierlein, Die Rezitationsdramen Senecas. Mit einem kritischexegetischen Anhang (Meisenheim am Glan, Hain, 1966; "Kritisches und Exegetisches zu den Tragödien Senecas. Bemerkungenan lässlich einer neuen Ausgabe", Philologus 113 (1969) 254-267; Prolegomena zu einer kritischen Ausgabe der Tragödien Senecas, Wiesbaden, 1984; Kritischer Kommentar zu den Tragödien Senecas, Stuttgat. Steiner, 1986; etc..

${ }^{17}$ Sénèque. Tragédies Tome I Hercule furieux. Les Troyennes. Les Phéniciennes. Médée. Phèdre, Texte établi et traduit par Léon Herrmann, Paris, Les Belles Lettres, 1925; Sénèque. Tragédies Tome II Oedipe. Agamemnon. Thyeste. Hercules sur l'Oeta. Pseudo-Sénèque. Octavie, Texte établi et traduit par Léon Herrmann, Paris, Les Belles Lettres, 1927. 
edición de las Tragedias, y despacha en fin en tres páginas lo que él llama "Sens des tragédies". No acertamos a comprender cómo la prestigiosa colección francesa ha podido publicar una cosa semejante. Por cierto: hasta en el distribución de los volúmenes se refleja la sombra omnipresente de Zwierlein: los dos en que se distribuían las tragedias en la ed. de Herrmann, ahora se aumentan a tres, conteniendo los dos primeros las ocho tragedias de paternidad no discutida ${ }^{18}$, uniendo en el volumen tercero la tragedia Hercules Oetaeus y la pretexta Octauia bajo el epígrafe "[PseudoSénèque]" 19 .

Por último, los años 2007 y 2009 nos sorprenden con una nueva edición crítica de las Tragedias de Séneca, la de Giancarlo Giardina ${ }^{20}$; y decimos que nos causó una sorpresa, o para expresarlo mejor, una muy grata sorpresa, porque era obra del mismo filólogo italiano con cuya edición de las Tragedias, editada en $1966^{21}$, habíamos trabajado ya hace ahora cuarenta años, la primera vez que investigamos sobre los dramas senecanos en una Tesis de licenciatura. Lo curioso es que ya aquella edición de hace cuarenta y cuatro años gozaba de enorme prestigio, a pesar de lo cual Giardina siguió trabajando incansablemente sobre las Tragedias, hasta llegar a esta edición del año pasado. No vamos a entrar, tampoco en este caso, en los aspectos ecdóticos; pero sí vamos a señalar una diferencia fundamental: en la edición de 1966, Giardina editaba el Hercules Oetaeus y la pretexta Octauia; en la de 2007-2009 ambas tragedias son excluidas. Coinciden, pues, los contenidos de los dos volúmenes con el de los vols. I y II de la edición de Chaumartin; y no sabemos si va a haber, como en el

${ }^{18}$ Sénèque.Tragédies TomeI Herculefurieux.Les Troyennes.Les Phéniciennes. Médée. Phèdre, Texte établi et traduit par François-Regis Chaumartin, Paris, Les Belles Lettres, 1996; Sénèque. Tragédies Tome II Oedipe. Agamemnon. Thyeste, Texte établi et traduit par François-Regis Chaumartin, Paris, Les Belles Lettres, 1999.

${ }^{19}$ Sénèque. Tragédies Tome III [Psudo-Sénèque] Hercule sur l'Oeta. Octavie, Texte établi et traduit par François-Regis Chaumartin, Paris, Les Belles Lettres, 1999.

${ }^{20}$ Lucio Anneo Seneca, Tragedie I Ercole, Le Troiane, Le Fenicie, Medea, Fedra, Edizione critica a cura di Giancarlo Giardina, Pisa - Roma, Fabrizio Serra Editore, 2007; Lucio Anneo Seneca, Tragedie II Edipo, Agamennone, Tieste, Edizione critica a cura di Giancarlo Giardina, Pisa - Roma, Fabrizio Serra Editore, 2009.

${ }^{21}$ L. Annaei Senecae Tragoediae, 2 vols., Bologna, Ed. Compositori, 1966. 
caso de la Colección Budé, un tercer volumen con las dos tragedias que también Giardina considera no pertenecientes a Séneca.

\section{El corpus de las Tragedias de Séneca: el problema de la autenticidad}

El problema de la autoría de las nueve tragedias de tema mítico y de la pretexta Octauia que nos ha conservado la tradición (aparentemente incompleta la tragedia Phoenissae), remonta ya a la comparación de su presencia en las dos familias de códices: sabido es que la tradición fundamentada en el códice Etrusco presenta nueve tragedias: Hercules, Troades, Phoenissae, Medea, Phaedra, Oedippus, Agamennon, Thyestes, Hercules, dando idéntico título a la tragedia que abre y a la que cierra el conjunto; mientras que la familia A ofrece no sólo cambios en los títulos y en el orden de las tragedias, sino que añade la pretexta Octauia: Hercules furens, Thyestes, Thebais [Phoenissae en E], Hyppolitus [Phaedra en E], Oedipus, Troas [Troades en E], Medea, Agamemnon, Octauia, Hercules Oetaeus.

En cuanto a la Octauia, la abundancia de argumentos que apoyan la negación de su autoría a Séneca hace que tal sea la posición mantenida desde hace muchos años por la generalidad de las opiniones, si bien sigue existiendo alguna obstinada defensa en el sentido contrario. Mucho más problemático resulta el tema de la paternidad senecana para Hercules Oetaeus, si bien los estudiosos y comentaristas más rigurosos de los últimos años defienden, con argumentos de índole variada, pero no de peso absoluto y contundente, que tampoco esta tragedia fue escrita por Séneca. En este contexto, la postura de Zwierlein pesa con fuerza: la Octauia, tragedia pretexta compuesta poco después de la muerte de Nerón, se colocaría en el arquetipo $A$ antes del Hercules Oetaeus, escrito a mediados del siglo II o en tiempos de Juvenal, después de Silio Itálico, según sostiene con firmeza Zwierlein, no dejando dudas en contra de la atribución a Séneca; siendo esto así, los títulos Hercules furens y Hercules Oetaeus serían un recurso para diferenciar las dos tragedias de Hércules, la auténtica de Séneca y la tardía, colocadas al principio y al final de los códices de las dos familias, pero con el único título de Hercules para ambas en el $E$.

Naturalmente, como ocurre casi siempre en la investigación sobre las Tragedias de Séneca, el tema no está zanjado por completo. Nuestra postura consiste en negar con firmeza la paternidad de Séneca para la Octauia, e igualmente, aunque no con la misma certeza y contundencia, para el 
Hercules Oetaeus. Esto nos plantea dos problemas fundamentales a la hora de concebir nuestro libro Séneca, Tragedias: el primero consiste en si debemos o no incluir ambas tragedias en nuestro estudio, cosa que nos inclinamos a hacer, si bien organizando el material de forma que resulte claro cuáles son las piezas de Séneca y cuáles las de autor desconocido. El segundo problema se encuentra ya, por ejemplo, en el título que Zwierlein puso a su edición oxoniense: L. Annaei Senecae Tragoediae Incertorum auctorum Hercules [Oetaeus] Octauia; ¿pero cómo se titulan las tragoediae auténticas? Está claro que Zwierlein, como todos los editores, prefiere los títulos de E, y elimina los títulos Thebais, Hyppolitus, Troas; ¿pero qué hacemos con Hercules furens? Zwierlein lo tiene claro, no admitiendo el adjetivo furens, que no sería en modo alguno senecano, y que además se aparta de la norma de Séneca de designar cada una de sus tragedias con un solo nombre propio, sin ningún tipo de adjetivación; Chaumartin, en cambio, sigue editando la obra como Hercule furieux; el último Giardina Hercules / Ercole. Por nuestra parte, después reflexionar mucho sobre el asunto, llamaremos exclusivamente Hércules a la tragedia indudablemente de Séneca, y Hércules en el Eta a la tragedia anónima.

\section{Representación, recitación, lectura de las Tragedias}

Un opúsculo de Gaston Boissier preguntaba en 1861, es decir, hace exactamente siglo y medio, si las tragedias de Séneca fueron leídas, recitadas o representadas. También nosotros incurrimos en ese tema, en concreto en 1973, en una de las primeras publicaciones de carácter filológico de A. Pociña ${ }^{22}$, alineándonos en el bando de los defensores de la lectura o recitación, y admitiendo la mínima probabilidad de que hubiesen sido representadas. No todas las argumentaciones que entonces pusimos en juego eran totalmente correctas; si hoy volviésemos a escribir aquel artículo, suprimiríamos muchas afirmaciones y añadiríamos otras tantas, para llegar con toda probabilidad al mismo resultado. En todo caso, dado que no vamos a entrar en el tema, que de todas formas tendremos que tratar en nuestro libro Séneca, Tragedias, recordaremos que: a) No existe información alguna antigua sobre la representación. b) Rara vez se representaron tragedias en tiempos de Séneca. c) No eran, en cambio, infrecuentes las

${ }^{22}$ A. Pociña, "Una vez más sobre la representación de las tragedias de Séneca", Emerita 41 (1973) 297-308. 
lecturas, en círculos privados o públicos, de obras poéticas, entre ellas de tragedias.

A la hora de hacer un planteamiento del tema, rechazamos de la forma más absoluta el presuponer que las Tragedias de Séneca no sean representables, así como seguirles dedicando comentarios y comentarios a la búsqueda de incoherencias y defectos dramáticos que conduzcan, como meta esencial, a la negación de la representatividad. Séneca escribió tragedias, y las escribió como tragedias. Pero eso no indica insoslayablemente que fueran representadas en su tiempo.

Por supuesto, argumentar a favor de la representación partiendo del hecho de que han sido representadas posteriormente, y que siguen siendo representadas en nuestro tiempo, y con éxito, resulta un argumento absurdo, carente de sentido: Florence Dupont comienza un interesante libro sobre el teatro de Séneca, que poseemos con una bellísima y muy amistosa dedicatoria suya ${ }^{23}$, con estas palabras: "Voici un livre d'actualité. Il est possible aujourd' hui de proposer une dramaturgie des tragédies de Sénèque parce que, depuis cinq ans à peine, les mises en scène se multiplient en France et en Suisse..." La justificación no puede ser más superficial, al igual que la nota que la acompaña, según la cual "l'école allemande" sigue afirmando que Séneca no es representable, mientras que "les Italiens" sostienen lo contrario. La verdad es que no se puede investigar de este modo, y pretender que se está haciendo filología seria.

Un motivo de reflexión, sobre todo para posibles investigadores e investigadoras de las Tragedias que no suelan aproximarse a otros teatros: en el año 1966, el gran novelista español Miguel Delibes, fallecido el año pasado, publica una de sus mejores novelas, Cinco horas con Mario, cuya parte más larga es el monólogo de una viuda que vela el cadáver de su marido y mantiene con él un diálogo de una sola voz en la noche anterior a darle sepultura. En el año 1979, se estrena en los teatros Cinco horas con Mario, interpretada por la excepcional actriz española Lola Herrera, obteniendo un éxito que perdurará, por los teatros de toda España, durante más de diez años. Y preguntamos: ¿alguien en su sano juicio estudiará esta novela de Delibes para llegar a la conclusión de que es una obra teatral, o como tal fue concebida y escrita por el gran novelista vallisoletano? No

${ }^{23}$ F. Dupont, Les monstres de Sénèque. Pour une dramaturgie de la tragédie romaine, Paris, Éditions Belin, 1995. 
olvidemos que nuestros estudios también son científicos, y como tales debemos plantearlos y defenderlos.

\section{El manido asunto de la datación de las Tragedias}

Cuando alguien se enfrenta a las Tragedias de Séneca con el propósito de estudiarlas, raro será que no se encuentre, antes o después, con el problema de la datación, ya sea del conjunto, ya de cada una de las piezas, posibilidad que dificulta en gran manera la tarea. El tema de la datación ya aparecía hace cerca de cuarenta años en una de nuestras publicaciones ${ }^{24}$; desde entonces hemos tenido que abordarlo en más de una ocasión, llegando siempre, a pesar de multiplicar nuestras consultas bibliográficas, a la conclusión de que no parece posible encontrar una datación incontrovertible y unánimemente aceptada. El tema, pues, resulta feo, controvertido, farragoso, insoluble, aburrido, cansino; pero es obvio que no vamos a poder evitarlo en nuestro libro Séneca, Tragedias. Teniendo en cuenta la abundante bibliografía que tenemos reunida sobre este asunto, y pensando que no es este lugar ni ocasión propicios para entrar en su debate, nos vamos a permitir mostrar aquí de qué manera lo aborda, de forma rápida e inteligente, resumiendo en una corta página lo que se sabe más o menos después de haber consultado cientos de teorías, nuestro querido amigo Giuseppe Gilberto Biondi, en una estupenda edición, de alta divulgación de las tragedias Medea y Fedra ${ }^{25}$ :

Problema más completo y discutido es el de la cronología (y diacronía) de las tragedias. Su composición podría referirse, diseminadamente, a todo el arco de Séneca (Herzog, 1928) o ser circunscrita a un momento particular como: 1) el exilio a Córcega (las tragedias habrían sido escritas y conservadas en un cajón a la espera de mejores tiempos); 2) el período neroniano, secundando la pasión por el género trágico de Nerón el filósofopedagogo podría haber escrito su obra poética en función educadora, señalando en los héroes positivos o negativos exempla respectivamente a imitar o a evitar (esta hipótesis podría ser apoyada por Tácito Ann. 14, 52); 3 ) el

${ }^{24}$ Cf. A. Pociña, "Finalidad político-didáctica de las tragedias de Séneca", Emerita 44 (1976) 279-301, esp. pp. 296-299.

${ }^{25}$ Lucio Anneo Seneca, Medea. Fedra, Premessa al testo, introduzione e note di Giuseppe Gilberto Biondi. Traduzione di Alfonso Traina, Milano, Rizzoli, 1989, p. 32. 
retiro de los últimos años a la vida privada que le habría permitido escribir, además de las últimas obras, también las tragedias, que en este caso no serían ya para sino contra Nerón. [...] La hipótesis más probable es que Séneca se haya hecho tragediógrafo para intentar, también por la vía de la palabra "medida", la educación o la "conmoción" del príncipe hacia el bien de la humanidad" $"$.

Naturalmente, se nos puede criticar que resumamos la cuestión problemática de la datación de las Tragedias con un pasaje ajeno que coincide muy bien con nuestras propias posturas, pero hemos de advertir que en los aspectos discutibles de la investigación senecana no es lo grave adoptar una postura determinada, sino enfrentarse de forma airada y contundente a posturas contrarias o ajenas.

\section{Ideología y finalidad de las Tragedias}

Las Tragedias de Séneca no son obra de oficio de una dramaturgo de profesión, ni de un escritor ocasional, aficionado, sino del pensador más importante de la Roma de su tiempo, y sin discusión digno de ser incluido en el número de los grandes pensadores universales. En consecuencia, tiene siglos de antigüedad la hermenéutica del teatro senecano basada en la comparación de la doctrina moral, filosófica, política, que se contiene y se transmite en las Tragedias y la que se manifiesta abiertamente, sin disfraz de ningún tipo y con una obvia finalidad pedagógica en las obras en prosa del Cordobés. En este sentido, existe una muy abundante bibliografía sobre el contenido doctrinal de los dramas, de obvia cimentación estoica, conforme a la doctrina del filósofo: en el primer apartado de nuestra exposición recordábamos ya el artículo bibliográfico de Aufstieg und Niedergang preparado por R. G. Tanner sobre el aspecto concreto "Stoic Philosophy and Roman Tradition in Senecan Tragedy" 27 , al que añadiríamos ahora, en la misma obra y volumen, el escrito por E. Lefevre sobre la ejemplificación por medio del Thyestes del significado filosófico de las Tragedias ${ }^{28}$. Resulta

${ }^{26}$ G. G. Biondi, cf. nota precedente, p. 32 s. La traducción del italiano es nuestra.

${ }^{27}$ R. G. Tanner, "Stoic Philosophy and Roman Tradition in Senecan Tragedy", ANRW II 32,2 (1985) 1100-1133.

${ }^{28}$ E. Lefevre, "Die philosophische Bedeutung des Seneca-Tragödie am Beispiel des Thyestes", ANRW II 32,2 (1985) 1263-1283. 
muy arriesgado hacer una selección de títulos destacados en tan amplia bibliografía, si bien nuestra particular visión de los mismos nos podría permitir recomendar, por lo que atañe a las Tragedias, los trabajos de Pratt $^{29}$, E. C. Evans ${ }^{30}$, J. C. García Borrón ${ }^{31}$, E. Lefèvre ${ }^{32}$ M. Cacciaglia ${ }^{33}$, T. G. Rosenmeyer ${ }^{34}$, H. M. Hine ${ }^{35}$; y sobre la ideología senecana en genral, las aportaciones recientes de B. Inwood ${ }^{36}$, J. Wildberger ${ }^{37}$.

Pero además de filósofo, Séneca ocupa un puesto de inmenso relieve en la política de su tiempo, como se ha dicho y estudiado mil veces, por ejemplo en una obra a nuestro modo de ver tan notable como la clásica de M. T. Griffin ${ }^{38}$. Entonces, hay que considerar la alta posibilidad de que nuestro autor haya compuesto sus tragedias en el período en que actuó primero como preceptor, después como ministro de Nerón, no le haya sido ajena la intención de servirse de su obra teatral para conducir por caminos adecuados el espíritu extraño y atrabiliario del emperador, que, además, adoraba el teatro. Hace un momento leíamos un breve pasaje en el que opinaba de este modo un analista tan fino de la producción trágica del Filósofo como es Biondi, y más arriba hemos aludido también a un trabajo nuestro de juventud, en el que defendíamos la existencia de una finalidad

${ }^{29}$ N. T. Pratt, "The Stoic Base for Senecan Drama”, TAPhA 79 (1948) 1-11.

${ }^{30}$ E. C. Evans, "A Stoic Aspect of Senecan Drama: Portraiture", TAPhA 81 (1950) 164-184.

${ }^{31}$ J. C. García Borrón, "El senequismo en el teatro de Lucio Anneo Séneca", RF 17 (1958) 65-70.

${ }^{32}$ E. Lefèvre, "Quid ratio possit? Senecas Phaedra als stoisches Drama", WS 3 (1969) 131-160.

${ }^{33}$ M. Cacciaglia, "L'etica stoica nei drammi di Seneca”, RIL 108 (1974) 78-104. 1989.

34 T. G. Rosenmeyer, Senecan Drama and Stoic Cosmology, Berkeley,

${ }^{35}$ H. M. Hine, "Interpretatio stoica of Senecan tragedy", en AA. VV., Sénèque le tragique. Huit exposés suivis de discussions, Vandoeuvres - Genève, Fondation Hardt, 2004, pp. 173-209.

${ }^{36}$ B. Inwood, Reading Seneca: Stoic philosophy at Rome, Oxford, Clarendon Pr. - New York, Oxford University Pr., 2005.

${ }^{37}$ J. Wildberger, Seneca und die Stoa: der Platz des Menschen in der Welt, Berlin, New York, de Gruyter, 2006. 1976.

${ }^{38}$ M. T. Griffin, Seneca. A Philosopher in Politics, Oxford, Clarendon Press, 
político-didáctica en las Tragedias ${ }^{39}$. Es una teoría que hemos seguido encontrando, en fechas anteriores y posteriores a la formulación de la nuestra, y sobre la que insistiremos de nuevo, también con planteamientos nuevos, en nuestro Séneca, Tragedias. Pero en la imposibilidad, una vez más, de profundizar sobre tema tan polémico, vamos a recordar un pasaje de la defensa de una postura semejante a la nuestra en un magnífico estudioso, editor y traductor italiano de los dramas senecanos, Giovanni Viansino, en esta ocasión en su versión original:

Centro focale delle tragedie è proprio l'ossessiva, realistica tematica del regno (...) e del potere, con riflessioni e rappresentazioni non aforistiche, ma tanto dirette, personali, esemplari quanto lo erano stati i precetti offerti a Nerone nei due libri dell'opera Della clemenza: a chi può essere ed è causa degli orrori che dilaniano una società, va indirizzato ogni possibile consiglio ad assumere comportamenti "paternalistici", razionali, stile liberale nelle relazioni interumane ${ }^{40}$.

\section{Reescrituras (solamente en los siglos XX y XXI)}

El tema de la tradición y pervivencia de las Tragedias de Séneca ha sido desde hace tiempo objeto de frecuente atención por parte de la investigación, cosa absolutamente natural si se tiene en cuenta su importancia en los teatros de todos los tiempos. Disponemos, pues, de muy importantes estudios generales sobre la pervivencia y el influjo de los dramas senecanos en el teatro europeo en general ${ }^{41}$, en los del Renacimiento ${ }^{42}$, en el teatro inglés elisabethiano, con especial atención a Shakespeare ${ }^{43}$, en el teatro

${ }^{39}$ A. Pociña, "Finalidad político-didáctica de las tragedias de Séneca", Emerita 44 (1976) 279-301, esp. pp. 296-299.

${ }^{40}$ G. Viansino, "Introduzione" a Seneca Teatro, Milano, Mondadori Editore, 2007 ( $1^{\mathrm{a}}$ ed. 1993), pp. 6-7.

${ }^{41}$ E. Lefevre (ed.), Der Einfluss Senecas aud das europäische Drama, Darmstadt, 2 vols., 1978.

${ }^{42}$ Cf. H. B. Charlton, The Senecan tradition in Renaissance tragedy, Manchester, 1946; J. Jacquot (ed.), Les tragédies de Sénèque et le théâtre de la Renaissance, Paris, 1964; P. Stachel, Seneca und das deutsche Renaissancedrama, New York, 1907 (1967); G. Braden, Renasissance tragedy and the Senecan tradition. Anger's privilege, Yale, 1985.

${ }^{43}$ Cf. N. E. Fleming, The influence of Seneca on Shakespeare, Liverpool, 
francés ${ }^{44}$, en dramaturgos concretos de los diversos teatros, cuya bibliografía sería prolijo y enojoso reproducir en este lugar, etc. Este aspecto, importantísimo desde el punto de vista de la historia del teatro universal, ocupará un puesto muy destacado en nuestro libro Séneca, Tragedias.

Ahora bien, más novedosa resultará la incorporación, en nuestra opinión absolutamente necesaria y siempre muy descuidada, de un amplio capítulo dedicado a la presentación y estudio de los hitos fundamentales de la pervivencia de las tragedias de Sénec en los teatros de habla española, tanto los de España como de las diversas naciones de América, en el italiano y en el portugués, en el que se incluyen tanto Portugal como Brasil y las naciones del Palop. En este campo de estudio, verdaderamente marginado (piénsese, por ejemplo, que en el famoso libro Antígonas de Georges Steiner no se cita ni una sóla Antígona en español o en portugués ${ }^{45}$, hemos estado trabajando intensamente en los últimos años y hemos publicado numerosos estudios (artículos y libros), al amparo del Proyecto de investigación subvencionado por el Ministerio de Educación titulado "El teatro latino y su influencia en los teatros europeos. I. Séneca" (años 2007-2009). Por otra parte, hemos intentado fomentar, en la medida de nuestras posibilidades, los estudios sobre la fortuna del teatro grecolatino en la historia del teatro, y en especial en el del siglo XX, coordinando obras colectivas como Medeas. Versiones de un mito desde Grecia hasta hoy ${ }^{46}$ (2002); Fedras de ayer y de hoy. Teatro, poesía, narrativa y cine ante un mito clásico $^{47}$ (2008); por último, un libro colectivo al que le tenemos un especial

1957; J. W. Cunliffe, The Influence of Seneca on Elizabethan Tragedy, London, Macmillan, 1893; F. L. Lucas, Seneca and Elizabethan Tragedy, Cambridge, 1922; A. L. Reimers, Seneca and the Elizabethan Tragedy, Stellenbosch, 1933; H. H. Sawler, The influence of Seneca on Elizabethan Tragedy, Dalhousie, 1937; R. R. Reed, Seneca and Elizabethan Tragedy, New York, 1947; J. R. Sutherland, Shakespeare and Seneca: a symbolic language for tragedy, Boulder, 1985; R. S. Miola, Shakespeare and Classical Tragedy. The Influence of Seneca, Oxford, Clarendon Press, 1992.

${ }^{44}$ E. Paratore, "Seneca tragico e la poesia tragica francese del Siècle d'or", StudUrb47 (1973) 32-60.

${ }^{45}$ G. Steiner, Antígonas, Trad. A. L. Bixio, Barcelona, Editorial Gedisa, 1991.

${ }^{46}$ A. López - A. Pociña (eds.), Medeas. Versiones de un mito desde Grecia hasta hoy, 2 vols., Granada, Universidad, 2002.

${ }^{47}$ A. Pociña - A. López (eds.), Fedras de ayer y de hoy. Teatro, poesía, narrativa y cine ante un mito clásico, Granada, Universidad, 2008. 
cariño, En recuerdo de Beatriz Rabaza. Comedias, tragedias y leyendas grecorromanas en el teatro del siglo $X X^{48}$, todas ellas obras colectivas en las que hemos contado con la importante y entusiasta aportación de filólogas y filólogos de Portugal.

En este aspecto, aunque de forma general, es decir, no referida exclusivamente a la pervivencia de las tragedias de Séneca, sino también a la de los tres grandes trágicos griegos, se mueve una parte de la investigación de las cuatro filólogas y los tres filólogos de España, Italia y Portugal que estamos realizando la obra sobre los cuatro grandes tragediógrafos de Grecia y Roma de que hablamos al principio y que ya demostramos al ser, el conjuntos los siete, editores y editoras del libro $O$ mito de Helena de Tróia à actualidade, publicado aquí, en Coimbra, en 1908; un libro que recoge los trabajos que presentamos en esta Universidad, en un memorable congreso celebrado en abril del año 2006.

Y, en este mismo orden de cosas, nos estamos preparando para celebrar el "Congreso Internacional sobre la Pervivencia de los Modelos Clásicos en Iberoamérica, España y Portugal", que tendrá lugar en la ciudad argentina de Mar del Plata, los días 23 al 27 de agosto de este año, al que nos mueve una inmensa ilusión, entre otras cosas porque contaremos con una selecta asistencia de colegas de España y de Portugal, representados por las dos Vicepresidentas del Congreso, por Portugal la Dra. Maria de Fátima Silva, por España la Dra. Aurora López, cabiéndole a Argentina, en representación de los paises americanos de habla española y portuguesa, la Presidencia, en la figura del Profesor de la Universidad de Mar del Plata Dr. Rómulo Pianacci.

${ }^{48}$ A. López - A. Pociña (eds.), En recuerdo de Beatriz Rabaza. Comedias, tragedias y leyendas grecorromanas en el teatro del siglo XX, Granada, Universidad, 2009. 